
allemande

\title{
1917 : l'année où la Discorde nationale dépassa les frontières de l'État grec
}

Anastasios Zografos

\section{(2) OpenEdition}

1 Journals

Édition électronique

URL : https://journals.openedition.org/allemagne/580

DOI : 10.4000/allemagne.580

ISSN : 2605-7913

Éditeur

Société d'études allemandes

Édition imprimée

Date de publication : 29 décembre 2017

Pagination : 381-392

ISSN : 0035-0974

\section{Référence électronique}

Anastasios Zografos, « 1917 : l'année où la Discorde nationale dépassa les frontières de l'État grec », Revue d'Allemagne et des pays de langue allemande [En ligne], 49-2 | 2017, mis en ligne le 29 décembre 2018, consulté le 21 mai 2021. URL : http://journals.openedition.org/allemagne/580 ; DOI : https:// doi.org/10.4000/allemagne.580 


\section{7: I'année où la Discorde nationale dépassa les frontières de l'État grec}

\section{- Anastasios Zografos*}

Quand la Grande Guerre éclata, la péninsule balkanique donnait l'impression d'une région où les peuples n'avaient pas encore réglé leurs comptes malgré les deux guerres balkaniques de 1912 et 1913. Les revendications des différentes nations de cette contrée conduisaient d'ailleurs, depuis un siècle, presque toujours à des tensions, voire très souvent, à la guerre.

En cet été 1914, la Grèce semblait ainsi prête à mener une nouvelle guerre pour réaliser sa Grande Idée ${ }^{(1)}$. Toutefois, le Premier ministre grec, Élefthérios Venizélos, décida de ne pas impliquer son pays dans un conflit périphérique malgré le traité de défense mutuelle que la Grèce avait signé avec la Serbie ${ }^{(2)}$.

Or, en peu de temps, la guerre se généralisa et la politique que l'État grec dut suivre face à ce conflit devint le sujet «chaud» qui occupa grandement la scène politique et la société grecque. Les deux forces politiques principales de l'époque en arrivèrent à entrer en conflit l'une contre l'autre; le pays connut alors des jours sombres, et se divisa en deux parties tandis que la crise interne grecque se diffusa en Europe.

Cette diffusion passa en effet autant par le biais des forces armées grecques «capturées» en automne 1916 et transférées à Görlitz que par la famille royale qui, ayant quitté la Grèce en été 1917, trouva refuge en Suisse. À la lumière des sources manuscrites de l'époque et surtout des carnets de guerre de deux officiers de la gendarmerie

* Docteur en histoire militaire, Université Paul-Valéry/Montpellier III; enseignant vacataire, Université de Chypre. Je voudrais remercier le professeur de l'Université Paul Valéry/Montpellier III, M. Hubert Heyriès, pour les corrections stylistiques apportées.

1 Grande Idée: idée nationaliste grecque née au XIX ${ }^{\mathrm{e}}$ siècle qui préconisait l'unification de toutes les contrées à populations grecques en un seul État dont le centre politique serait la ville de Constantinople.

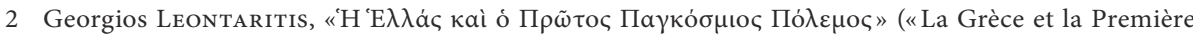

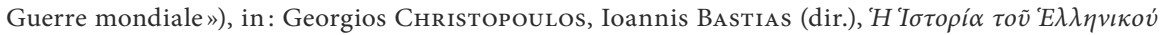
É $\theta$ vov (Histoire de la Nation grecque), Athènes, Éditions d’Athènes, 1978, vol. 15, p. 15. Tous les titres grecs sont traduits en français par l'auteur de l'article. 
qui suivirent les forces armées grecques en Allemagne, nous tenterons d'analyser cette diffusion de la Discorde nationale grecque en Europe.

Ces deux carnets sont sans doute des sources très importantes non seulement en raison de leur contenu mais aussi du fait de leur rareté. Ces manuscrits rédigés par des officiers grecs décrivent la «captivité » et la façon avec laquelle la Discorde nationale toucha la vie des troupes grecques hors de la Grèce. Quant à leur rareté, il suffit de souligner que de nombreux carnets similaires furent détruits par leurs possesseurs durant cette période ${ }^{(3)}$. Au cours de cette crise majeure qui vit les ennemis politiques des uns et des autres s'épurer mutuellement, la possession de tels carnets était dangereuse non seulement pour leurs propriétaires mais aussi pour les personnes qui y étaient citées.

Quelle fut donc cette crise politique grecque du début du $\mathrm{XX}^{\mathrm{e}}$ siècle? Comment dépassa-t-elle les frontières du pays? Quelle fut son évolution au-delà de la Grèce? Et, de quelle façon toucha-t-elle la vie des forces armées grecques, celle des civils et celle des autorités allemandes?

\section{Une crise politique majeure qui se diffuse à l'étranger}

Au début de la Grande Guerre, la politique de neutralité suivie par Athènes sembla coïncider avec celle préférée par les Alliés. Ces derniers cherchaient à imposer à tous les pays balkaniques la neutralité ou bien à les impliquer dans leur guerre contre les Puissances centrales. Mais peu à peu, les Alliés et surtout les Britanniques commencèrent à chercher à faire entrer en guerre la Grèce à leurs côtés ${ }^{(4)}$. Or, le pays était divisé sur ce point.

Pour Venizélos, la neutralité grecque n'était qu'une politique provisoire en attendant le moment favorable d'une entrée en guerre aux côtés des Alliés. Convaincu que, malgré tout, l'Angleterre serait toujours la Grande puissance qui contrôlerait la Méditerranée orientale, il visait à se rapprocher de Londres. Au contraire, le roi Constantin trouvait que la Grèce devait suivre une politique de neutralité puisque, pour lui, l'Allemagne serait victorieuse ${ }^{(5)}$.

Certes, les deux positions différentes traduisaient surtout deux conceptions contradictoires au sujet du modèle politique que la Grèce devait suivre à l'avenir. Et si Élefthérios Venizélos avait choisi celui «de la justice, de la civilisation et de la paix» ${ }^{(6)}$, le roi Constantin se tourna plutôt vers le modèle allemand. Ayant étudié en Allemagne, admirateur de la monarchie allemande et mari de la sœur du Kaiser, le roi de la Grèce se sentait en effet si proche de Berlin que choisir la neutralité de son pays était une façon de soutenir sa belle Allemagne ${ }^{(7)}$.

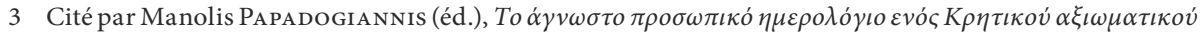
(Le carnet inconnu d'un officier vénizéliste), Thessalonique, Manoles Papadogiannis, 1990, p. 11.

4 Pour plus d'informations, G. Leontaritis, «La Grèce et la Première Guerre mondiale ( note 2), p. 15-18.

5 Ibid.

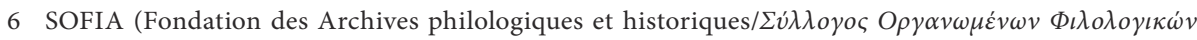

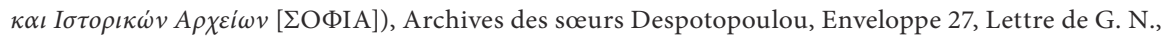
22 octobre 1918, p. 1.

7 Loukianos Hassiotis, «Greece», in: Daniel Ute, Peter Gatrell, Oliver Janz et al., 1914-1918 online. International Encyclopedia of the First World War, Freie Universität Berlin, Berlin, 10 août 2014. Consulté en ligne; lien: http://dx.doi.org/10.15463/ie1418.10043. 
En peu de temps, cette opposition entre les deux hommes se transforma en guerre civile. La première étape se déroula durant la campagne alliée dans les Dardanelles. Celle-ci, ayant comme objectif la ville de Constantinople, secoua les esprits en Grèce. La presse locale rappela ainsi au monde politique que la place de la Grèce n'était pas celle de spectateur et de laudateur de l'hérö̈sme des autres ${ }^{(8)}$. Fin février 1915, le Premier ministre, convaincu que l'heure de l'entrée de la Grèce en guerre était arrivée et s'étant assuré de l'appui des Anglais, annonça sa décision au roi. Mais à l'issue des discussions au sein du Conseil royal, discussions qui furent intenses, le roi Constantin informa Venizélos que la Grèce devait rester neutre et il le fit démissionner ${ }^{(9)}$.

Dans un tel contexte, les élections de juin 1915 prirent la forme d'un référendum au sujet de la participation ou non du pays à la guerre. Le résultat montra, en fait, que le peuple grec soutenait Élefthérios Venizélos. La crise était loin d’être finie.

Trois mois plus tard, le vote de la Chambre ratifia la proposition du Premier ministre validant l'entrée en guerre de la Grèce pour aider la Serbie. Le jour même, Venizélos se rendit au palais mais il se heurta encore une fois au refus du roi, et fut contraint, de nouveau, à la démission ${ }^{(10)}$. Début décembre 1915, de nouvelles élections eurent lieu. Mais comme le parti vénizéliste refusa d'y participer, l'Assemblée élue ne fut composée que des députés du parti antivénizéliste. Désormais, la politique suivie par Athènes serait celle imposée par le roi ${ }^{(11)}$.

À l'aube de l'année 1916, la crise politique toucha aussi la société et l'armée du pays. De nombreuses manifestations pro-royalistes occupèrent la rue, diabolisant Élefthérios Venizélos ${ }^{(12)}$ et persécutant ses partisans ${ }^{(13)}$. Des troupes clandestines royalistes, composées de soldats démobilisés, s'organisaient ici et là et terrorisaient le pays tout entier et surtout ces ennemis politiques ${ }^{(14)}$. De plus, tous ceux qui, dans l'entourage du roi, exprimaient la moindre sympathie envers l'homme politique crétois ou le moindre doute sur la politique suivie par le palais furent immédiatement expulsés ${ }^{(15)}$.

Dans ce climat délétère, les officiers fidèles à Élefthérios Venizélos ne purent pas rester passifs. Fin août 1916, à Salonique, ils se soulevèrent en faveur de l'ex-Premier

8 «La voie du destin», Empros, n 6584 (16 février 1916), p. 1. Tous les titres des articles des journaux grecs sont, eux aussi, traduits par l'auteur de l'article.

9 G. Leontaritis, «La Grèce et la Première Guerre mondiale» (note 2), p. 18-22; Angeliki Dima-Dimi-

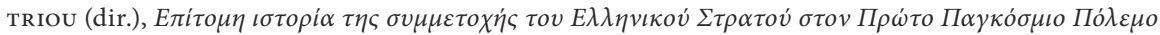
1914-1918 (Histoire de la participation de l'armée grecque à la Première Guerre mondiale 1914-1918), Athènes, Direction de l'histoire de l'armée grecque, 1993, p. 17-29.

10 Ibid.

11 Ibid.

12 FNREEV (Fondation nationale de la Recherche et des Études «Élefthérios Venizélos »/E $\theta v \iota \kappa o ́$ 'I $\delta \rho v \mu \alpha$

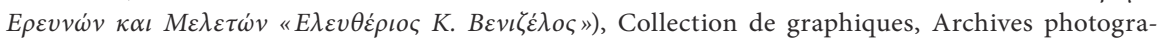
phiques, Enveloppe 1, n 166-149.

13 Ibid., Collection d'archives, Archives d'Em. N. Georgakakis, Enveloppe 1, Demande adressée au consul de la Grande-Bretagne, 8 avril 1917.

14 Musée Benakis, Archives d’Élefthérios Venizélos, Enveloppe 413, Lettre quant à l'attaque des troupes clandestines antivénizélistes contre des magasins des vénizélistes de Prévéza, 23 juin 1916.

15 ELIA-MIET (Archives historiques et littéraires grecques - Institution éducative de la Banque natio-

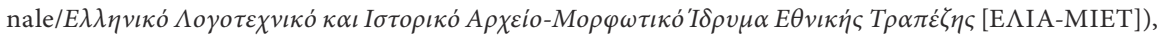
Archive de la Famille de Melas, Georgios M. Melas, Mon carnet personnel, 1916-1917, p. 1-7. 
ministre grec, ce qui provoqua, les jours suivants, la division du pays ${ }^{(16)}$. Le 9 octobre 1916, l'homme politique crétois se rendit dans la ville et y fut triomphalement accueilli par la population locale ${ }^{(17)}$. Se mettant à la tête du soulèvement militaire, il fonda son propre gouvernement provisoire. Désormais, la Grèce avait deux centres politiques et deux gouvernements. L'un à Athènes où prédominaient le roi et ses fidèles favorables à la neutralité. L'autre à Salonique où Élefthérios Venizélos avec le soutien des forces armées locales se préparait à soutenir les Alliés.

Toutefois, en peu de temps, cette Discorde nationale finit par intéresser l'Europe. De quelle manière?

Tout commença en août 1916 lorsque des troupes des Empires centraux reçurent l'ordre du Quartier général allemand de progresser à l'intérieur de la Macédoine orientale $^{(18)}$. Or, dans le même temps, les forces grecques suivirent les ordres de leur gouvernement athénien de rester passives ${ }^{(19)}$, et quittèrent, sans se battre, leurs forts en se repliant sur le siège de chaque Division ${ }^{(20)}$. En l'espace de quelques jours, les troupes étrangères occupèrent ainsi entièrement la Macédoine orientale tandis que les civils se hâtaient de trouver refuge dans des grandes villes pour éviter les persécutions bulgares $^{(21)}$.

Les jours suivants, la situation s'aggrava. Le 10 septembre 1916, l'envoyé du Quartier général allemand ${ }^{(22)}$ informa le commandant en chef du IV corps d'armée que les troupes grecques devaient prendre la route vers le nord occupé par les Bulgares pour éviter toute collaboration avec les Alliés. L'officier grec, n’obtenant pas de réponses à ses messages adressés à Athènes, des messages qui demandaient l'autorisation de transférer ses hommes en Grèce du Sud, décida alors de proposer à Berlin, par le biais du commandant von Schweinitz, de se rendre aux Allemands ${ }^{(23)}$.

Pourtant, dans la nuit, le chef du IV e corps tenta de sauver ses hommes de leur «capture», en vain ${ }^{(24)}$. Le lendemain, le commandant allemand informa Ioannis Chatzopoulos que sa proposition avait été acceptée par le généralissime Hindenburg. Les deux hommes se mirent d'accord pour transférer en Allemagne les forces grecques de l'armée de terre et de la gendarmerie, munies de leur armement et de leurs munitions. Ces forces y vivraient jusqu'à la fin de la Grande Guerre avec le statut d'invités du

16 A. Dima-Dimitriou (dir.), Histoire de la participation (note 9), p. 87-91; G. Leontaritis, «La Grèce et la Première Guerre mondiale» (note 2), p. 36-38.

17 «Thessalonique en état d'alerte», Macédoine, 27 septembre 1916, n 1725, p. 1-3; FNREEV, Collection photographique, Archive d'Élefthérios Venizélos, Enveloppe 3, 1916, nº 014-1813.

18 En effet, elles l'avaient déjà envahie depuis fin mai 1916, occupant le fort de Rupel. A. DimA-DimiTRIOU (dir.), Histoire de la participation (note 9), p. 73-77.

19 Polychronis Enepekidis, $\Delta o ́ \xi \alpha \kappa \alpha \iota \Delta \iota \chi \alpha \sigma \mu o ́ \varsigma$ (La Gloire et la Discorde nationale), Athènes, Éditions Zacharopoulos, 1992, p. 492-498.

20 Rares furent les cas des officiers grecs qui ont résisté. Gerasimos Alexatos, Les Grecs de Görlitz, Thes-

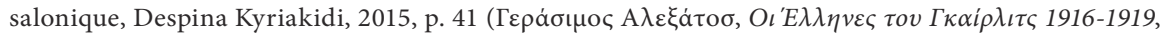

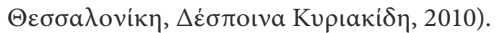

21 Voir entre autres le témoignage d'Ioannis Papadakis, M. Papadogiannis (éd.), Le carnet (note 3), p. 39-50.

22 C'était le commandant von Schweinitz.

23 G. Alexatos, Les Grecs (note 20), p. 56-57.

24 Ioannis Chatzopoulos tenta de transférer ses hommes au sud du pays par le biais des navires anglais. 
Kaiser et non celui de prisonniers de guerre ${ }^{(25)}$. Mais dorénavant, ce fut à Görlitz, la ville d'accueil choisi par le Quartier général allemand que la crise politique grecque prit toute son ampleur en raison du fait que des troupes vénizélistes côtoyaient des officiers et des soldats fidèles à leur roi, des militaires qui semblaient même contents d'avoir capitulé face aux Allemands ${ }^{(26)}$.

L'évolution de la situation en Macédoine choqua la Grèce entière et fut considérée comme une trahison du roi ${ }^{(27)}$. La presse vénizéliste publia des articles enflammés ${ }^{(28)}$. Les Allemands propageaient l'idée que les Grecs avaient auto-capitulé( ${ }^{(29)}$. Quant aux Alliés, les officiers en poste en Orient exprimèrent des commentaires amers sur les événements d'automne 1916 en proie à une impression horrible ${ }^{(30)}$.

Aussi, le général Sarrail ordonna aux navires français de bloquer le Pirée. En novembre 1916, les Alliés essayèrent une fois de plus de trouver un compromis avec le roi Constantin. Ce dernier donnait en effet l'impression d'être prêt à coopérer avec eux. On finit par s'entendre pour retirer les troupes grecques de Thessalie et remettre le matériel de guerre grec aux Alliés à la condition que ces derniers respectent la neutralité du pays sous tutelle royale et n'encouragent pas le gouvernement vénizéliste à s'étendre dans le sud ${ }^{(31)}$.

Ce fragile compromis ne tarda pas à échouer. Le gouvernement d'Athènes refusa en fait de remettre aux Alliés le matériel de guerre. Aussi, le $1^{\text {er }}$ décembre, des forces alliées débarquèrent au Pirée. Et une bataille éclata entre celles-ci et des troupes fidèles au roi ${ }^{(32)}$. Au même moment, des royalistes attaquèrent les vénizélistes; des pillages, des arrestations et des meurtres eurent lieu ${ }^{(33)}$.

La bataille d'Athènes et les Noémvriana choquèrent alors les Alliés et accélérèrent la démission du roi. Durant les mois suivants, un climat de tension et de menaces plana en Grèce. Aussi, début juin 1917, le roi Constantin, à la suite d'un mémorandum des Alliés, quitta son trône. La famille royale partit pour l'Italie et, le 15 juin 1917, gagna la Suisse ${ }^{(34)}$.

Dorénavant, la Grèce entière put prendre part à la guerre sous la direction d'Élefthérios Venizélos qui, de nouveau, se retrouva au pouvoir. Toujours est-il que l'arrivée

25 G. Alexatos, Les Grecs (note 20), p. 49-76; A. Dima-Dimitriou (dir.), Histoire de la participation (note 9), p. 98-105; M. Papadogiannis (éd.), Le carnet (note 3), p. 51-63.

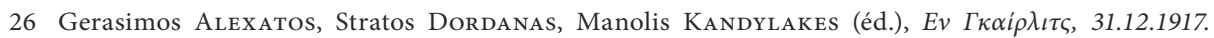

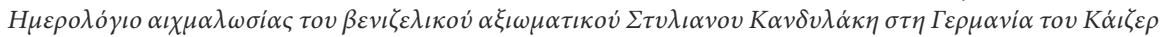
(À Görlitz, 31 décembre 1917. Carnet de captivité de l'officier vénizéliste Stylianos Kandylákes dans l’Allemagne du Kaiser), Thessalonique, Éditions Kiriakidis, 2014, p. 15.

27 ELIA-MIET, Archive de la Famille de Melas, Georgios M. Melas, Mon carnet personnel, 1916-1917, p. 1-7; «Les traîtres d'Athènes démasqués», Macédoine, $\mathrm{n}^{\circ} 1748$ (20 octobre 1916), p. 3.

28 Voir la presse vénizéliste du septembre 1916.

29 G. Alexatos, Les Grecs (note 20), p. 73-76.

30 Ibid.

31 Voir aussi G. Leontaritis, «La Grèce et la Première Guerre mondiale» (note 2), p. 41-42.

32 Ibid.; A. Dima-Dimitriou (dir.), Histoire de la participation (note 9), p. 129-138.

33 Ces événements sont aussi connus sous le nom de Noémvriana. Voir aussi G. Leontaritis, «La Grèce et la Première Guerre mondiale» (note 2), p. 41-42.

34 Ibid., p. 42-46. 
du roi Constantin en Suisse et la présence en Allemagne, depuis octobre 1916, d'un corps d'armée profondément antivénizéliste furent les vecteurs par lesquels la Discorde nationale pénétra en Europe. Mais sous quelle forme? Les antivénizélistes continuèrent-ils à tenter d'influencer le cours des événements en Grèce?

\section{La Discorde nationale en Europe}

Dès le début de leur nouveau cantonnement à Görlitz et jusqu'à la fin de la guerre, les troupes grecques, fantassins et gendarmes, s'affrontèrent entre elles sur le plan politique. Ce conflit prit même la forme d'une véritable guerre civile opposant d'un côté, une grande majorité royaliste et, de l'autre, une petite minorité vénizéliste. Ainsi, loin de la Grèce, ces "prisonniers" grecs ne tardèrent pas à transférer la crise politique grecque en Allemagne, ce qui obligea même parfois les autorités politiques locales à s'en mêler.

À partir d'octobre 1916, à l'arrivée des troupes grecques à Görlitz après un long voyage à travers les Balkans ${ }^{(35)}$, le contexte devint désastreux pour les officiers, les soldats et les gendarmes vénizélistes. Ces derniers, considérés par leurs ennemis politiques comme des hommes dangereux pour le corps d'armée et pour l'Allemagne, ne menèrent pas en Silésie la vie que des invités du Kaiser auraient dû mener. Les officiers royalistes, qui avaient même fondé leur propre association depuis février $1917^{(36)}$, firent leur possible pour susciter la méfiance des autochtones et des autorités locales envers les vénizélistes qui finirent par être considérés comme de véritables dangers publics. À peine un mois après son arrivée à Görlitz, l’officier vénizéliste Ioannis Papadakis écrivit ainsi dans son carnet de guerre:

"L'adjudant-chef Cristopoulos, qui occupe une chambre chez la tante de ma propriétaire ${ }^{(37)}$, lui a dit de faire attention parce que je suis vénizéliste et, donc, ennemi de l'Allemagne. J'ai aussi remarqué que des policiers allemands me suivent. Il me semble que mes camarades bien-aimés n’ont pas tardé à me présenter, ici également, comme un être dangereux.

Ma propriétaire m'a dit qu'il faut que je fasse attention parce que des policiers me suivent et que souvent ils lui demandent des informations sur moi. De plus, ils lui ont dit de m'espionner pour voir ce que je fais dans ma chambre.

Elle m'a dit qu'elle est socialiste et qu'elle hait le régime. Est-ce vrai ou l'avait-elle dit pour voir ma réaction? Soyons aux aguets!

[...]

Des policiers allemands [continuent à] me surveiller systématiquement et activement. Ma propriétaire a commencé à s'inquiéter et à avoir peur. Je remercie mes bons camarades qui m’ont dénoncé comme personne très dangereuse aux autorités allemandes » ${ }^{(38)}$.

Aussi, la vie des officiers et des soldats vénizélistes ne cessa de s'aggraver, ce qui rendit encore plus tendues leurs relations avec les royalistes. Ces derniers menèrent auprès des soldats et des gendarmes originaires soit de la diaspora grecque soit de

35 Sur le trajet que les «capturés» grecs ont suivi en automne 1916, G. Alexatos, Les Grecs (note 20), p. 78.

36 Alexatos/Dordanas/Kandylakes (éd.), À Görlitz (note 26), p. 80-81.

37 À Görlitz, les officiers grecs se sont installés au-delà du camp de concentration, chez des autochtones. Voir aussi G. Alexatos, Les Grecs (note 20), p. 87.

38 M. Papadogiannis (éd.), Le carnet (note 3), p. 72-74. Extrait traduit par l'auteur de l'article. 
la Crète une intense propagande antivénizéliste quotidiennement ${ }^{(39)}$. Pour ce faire, le Quartier général du corps ordonna de muter des officiers fidèles au roi dans des bataillons jugés dangereux en raison de l'origine géographique des hommes. Le bataillon de la gendarmerie, par exemple, fut l'une de ces unités. Elle devait en effet être épurée politiquement grâce à l'implantation d'un certain nombre d'officiers et sous-officiers royalistes au sein d'une majorité d'hommes originaires de la Crète et des régions de la Nouvelle Grèce $e^{(40)}$.

Par ailleurs, les vénizélistes - soldats, gendarmes et officiers - subirent des peines disciplinaires sévères pour des faits insignifiants, en raison tout simplement de leurs opinions politiques ou de leur origine crétoise ou de la Nouvelle Grèce $e^{(41)}$. Parfois aussi, il suffisait de déformer ou de rapporter les paroles d'un vénizéliste formulées à l'occasion d'une discussion privée. Le capitaine de la gendarmerie Stylianos Kandylákes fut ainsi puni à quarante-cinq jours d'emprisonnement pour avoir insulté le roi Constantin selon l'acte de l'accusation ${ }^{(42)}$.

En outre, les vénizélistes finirent par souffrir d’un isolement pénible, car la peur de subir des sanctions pour ne serait-ce qu'avoir bu un café avec l'un d'eux poussa le plus grand nombre d'hommes à ne plus les fréquenter. Et, cet isolement profond, dur à supporter durant cette longue période, devenait en fait encore plus douloureux durant les fêtes; ainsi, le moral des officiers vénizélistes s'en ressentit et beaucoup devinrent des loques humaines. L'officier de la gendarmerie Ioannis Papadakis décrivit dans son carnet de guerre ses sentiments le jour de Noël de 1916:

«Les officiers m'évitent soigneusement. Ils ont peur de m’approcher parce qu'ils peuvent être accusés de vénizélisme et à cause de cela, ainsi, tomber en disgrâce... Enfermé dans ma chambre, comme en quarantaine pour la peste, je passe de sombres journées.

Le 25 décembre, j’ai passé la journée comme un prisonnier. Cet isolement m’a fait et me fait toujours penser tout le temps à ma pauvre famille et pleurer pour elle. Le fait d'être seul et de ne pas avoir quelqu'un qui pourrait me réconforter, fait de moi littéralement un enragé. Je suis là, loin de mes enfants et sans avoir aucune nouvelle de ma famille depuis le 5 août, date à laquelle je suis parti de chez moi» ${ }^{(43)}$.

Quelques mois plus tard, lorsque le changement de gouvernement à Athènes accrut le fanatisme des royalistes, les officiers vénizélistes furent même exclus des lieux de rencontre et de distraction réservés à la troupe. À partir du début juillet 1917, le commandant en chef du IV ${ }^{e}$ corps d'armée interdit ainsi aux officiers vénizélistes de se rendre aux clubs des officiers de la ville pour déjeuner. Ces derniers furent alors obligés de déjeuner seuls ${ }^{(44)}$.

39 Les Crétois et les Grecs en provenance autant de la diaspora que de la Nouvelle Grèce furent, dans leur majorité, vénizélistes.

40 Conformément à la rhétorique de l'époque, la Vieille Grèce engloba tous les territoires qui constituaient le Royaume grec à la fin de la Révolution de 1821. La Nouvelle Grèce fut celle des territoires annexés au début du $\mathrm{xx}^{\mathrm{e}}$ siècle, à la fin des deux guerres balkaniques. M. PAPAdogiannis (éd.), Le carnet (note 3), p. 73-74.

Ibid.; Alexatos/Dordanas/Kandylakes (éd.), À Görlitz (note 26), p. 80-81.

42 Ibid., p. 17-18, 143.

43 M. Papadogiannis (éd.), Le carnet (note 3), p. 74. Extrait traduit par l'auteur de l'article.

Alexatos/Dordanas/Kandylakes (éd.), À Görlitz (note 26), p. 18. 
D’un autre côté, les insultes et les accusations d'antipatriotisme et de lâcheté tombèrent sur les vénizélistes. Les soldats qui étaient originaires de Crète ou dont le nom de famille avec la désinence «-akis» trahissait la provenance crétoise furent presque quotidiennement insultés par les officiers royalistes ${ }^{(45)}$. D’autres, y compris des officiers, reçurent des lettres anonymes outrageantes. Le 7 juin 1917, un certain Costas, dans une lettre adressée au colonel Synaniotis, écrivit alors avec une profonde ironie que le traître crétois, Élefthérios Venizélos, proposerait à la décoration l'officier grec, aux côtés d'autres lâches; mais pour montrer son manque de respect à l'égard de son colonel, il écrivit à la fin de la lettre: «Je t'emmerde» ${ }^{(46)}$.

Dans ce climat, la tension entre les deux camps politiques grecs en Allemagne devint rapidement si vive que l'on finit par se provoquer en duel et que l'on était prêt à en venir aux mains en pleine rue ${ }^{(47)}$. Ce furent les officiers royalistes qui se montrèrent les plus violents et exprimèrent très souvent une hostilité sans réserve envers les vénizélistes. Un sous-lieutenant royaliste n'hésita pas ainsi, en pleine rue, à battre un commandant pour des raisons politiques ${ }^{(48)}$. Un jeune sous-officier réserviste, apprenant l'acte d'accusation à l'encontre du capitaine de la gendarmerie Stylianos Kandylákes, l'interpella pour lui régler son compte à la sortie du club des officiers de Görlitz ${ }^{(49)}$. S’agissait-il d'un ordre de la part de l'un de ses supérieurs? Fut-il affecté par la "malhonnêteté» de son camarade? Était-il prêt à prouver sa fidélité au roi en tuant ou en se faisant tuer par un vénizéliste? Dans tous les cas, la réaction de ce jeune sous-officier de l'armée grecque montrait clairement que la Discorde nationale perturbait profondément les esprits des Grecs hors de Grèce.

Finalement à la fin 1917, après une longue période au cours de laquelle ils vécurent menacés par leurs supérieurs royalistes d'être emprisonnés ${ }^{(50)}$, des officiers et sous-officiers vénizélistes, autant de l'armée de terre que de la gendarmerie, furent arrêtés par les autorités allemandes de Görlitz. Vingt-trois officiers et sous-officiers grecs, invités par le commandant militaire de la ville de Görlitz au club des officiers de la Handelskammer, apprirent par le sous-colonel Rhazen que le IV e corps d'armée grec avait demandé leur arrestation pour des raisons politiques. Ils se trouvaient dorénavant à la disposition du ministère prussien de l'Armée sans possibilité de résister car un bataillon allemand, placé à la porte du club, était prêt à leur tirer dessus sur son ordre. Ils rendirent alors leurs armes tout en gardant leur épée ${ }^{(51)}$. Par la suite, ils furent conduits à la gare sous les regards réjouis de leurs camarades royalistes et montèrent dans le

45 Ibid., p. 81.

46 Musée Benakis, Archives d’Élefthérios Venizélos, Enveloppe 84, Lettre insultante anonyme adressée à Synaniotis, Görlitz, 7 juin 1917, p. 1.

47 Ibid., Demande de Synaniotis adressée au IV corps d'armée, Görlitz, 15 août 1917, n 951, p. 1; ibid., Enveloppe 354, Rapport d'Ioannis Papadakis quant à la vie des vénizélistes à Görlitz, Athènes, 14 février 1919, p. 3.

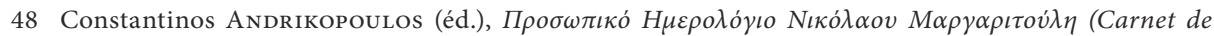
guerre de Nicolaos Margaritoulis), 2007-2009), consulté en ligne. Lien: http://paradoxon-klangor chester.de/paramithi/arthra/kato-o-polemos.html Alexatos/Dordanas/Kandylakes (éd.), À Görlitz (note 26), p. 17-18. M. Papadogiannis (éd.), Le carnet (note 3), p. 77-87. Alexatos/Dordanas/Kandylakes (éd.), À Görlitz (note 26), p. 82-83. 
train pour Werl en Westphalie ${ }^{(52)}$. Là, rejoints par treize autres camarades vénizélistes les jours suivants, ils furent enfermés dans un monastère de moines franciscains à côté de vingt-cinq officiers polonais ${ }^{(53)}$. Isolés du gros des troupes et assimilés à des prisonniers de guerre, les vénizélistes connurent, jusqu'à la fin de la Grande Guerre, une vie encore pire que celle menée en Silésie à cause de leurs opinions politiques.

À Werl, dès leur arrivée, les officiers durent rendre leur épée. Ils souffrirent d'une nourriture insuffisante et si pitoyable que les colis de vivres envoyés de Maastricht et parfois de Silésie leur furent indispensables pour survivre. La correspondance avec leurs camarades à Görlitz leur fut interdite et les sorties de leurs cellules dépendirent de leur comportement. Ils vécurent aussi dans le plus grand isolement car leurs coprisonniers polonais reçurent l'ordre du commandant de la prison de ne pas les fréquenter. Ce dernier les considérait en effet comme des ennemis de l'Allemagne et dans ses ordres il n'hésitait pas à rappeler que leur emprisonnement avait été ordonné par le commandant en chef de leur corps d'armée pour de graves délits tandis que les officiers polonais étaient des amis de Berlin. Et pour rendre la vie de ces ennemis politiques encore plus difficile, les royalistes de Görlitz refusèrent de leur consentir la visite d'un prêtre au monastère Exerzitienhaus der Franziskaner ${ }^{(54)}$.

En outre, les efforts des prisonniers de Werl pour améliorer leurs conditions de vie n'eurent aucun résultat. Les plaintes des vénizélistes adressées aux autorités grecques et allemandes tombèrent dans le vide. De même, leurs rapports adressés au consul hollandais ne changèrent ni leur statut ni leurs conditions de vie. Au contraire, la dizaine ou plus de rapports envoyés entre janvier et novembre 1918 ne fit que renforcer la méfiance du commandement de la prison à leur égard et à durcir leur condition d'emprisonnement ${ }^{(55)}$.

En effet, les trente-six officiers vénizélistes étaient désormais considérés comme prisonniers de guerre d'un pays ennemi ${ }^{(56)}$. Dans ce climat, leur vie devint de plus en plus invivable et les chances de survivre diminuèrent. En fait, les mesures prises par le gouvernement grec à l'encontre des ressortissants allemands de Grèce provoquèrent des représailles des autorités allemandes envers tous les vénizélistes qui se trouvaient en Allemagne et envers les officiers grecs de Werl. Une décision prise par le gouvernement de Berlin interdit ainsi de leur remettre les colis de vivres. L'un des prisonniers de Westphalie ne put s'empêcher d'écrire avec ironie dans son carnet: «Vive la civilisation! » ${ }^{(57)}$.

52 Ibid., p. 84; Musée Benakis, Archives d'Élefthérios Venizélos, Enveloppe 354, Rapport d'Ioannis Papadakis quant à la vie des vénizélistes à Görlitz, Athènes, 14 février 1919, p. 3.

53 Voir aussi G. Alexatos, Les Grecs (note 20), p. 169-176.

54 Alexatos/Dordanas/Kandylakes (éd.), À Görlitz (note 26), p. 87-88, 100-104.

55 Ibid., p. 80-115.

56 Fin juin 1917, Élefthérios Venizélos, prenant de nouveau le pouvoir à Athènes, déclara la guerre aux Puissances centrales. En effet, la Grèce vénizéliste entra dans la guerre aux côtés des Alliés fin novembre 1916. Dorénavant, le Premier ministre grec ne fit qu'impliquer son pays entier dans cette guerre mondiale. Voir entre autres, A. Dima-Dimitriou (dir.), Histoire de la participation (note 9), p. 169-172.

57 Alexatos/Dordanas/Kandylakes (éd.), À Görlitz (note 26), p. 104-105. 
Dans le même temps, les royalistes se rangeaient du côté de l'Allemagne, conformément à la politique germanophile menée par le roi Constantin depuis le début de la guerre. Or ce dernier, exilé en Suisse, se trouvait plus proche et il espérait profiter des forces armées grecques installées en Allemagne pour renverser le gouvernement vénizéliste et remonter en triomphateur sur son trône ${ }^{(58)}$.

Le 30 novembre 1917, le consul grec de Berne informa ainsi le ministre grec des Affaires étrangères et le consul grec de Paris que, selon ses informations, le commandant en chef du IV ${ }^{\mathrm{e}}$ corps d'armée, avec d'autres officiers grecs, avait rendu visite au roi Constantin en Suisse ${ }^{(59)}$. De fait, trois mois plus tard, deux officiers royalistes, en provenance de Görlitz, furent arrêtés à Athènes et condamnés à mort après être passés en cour martiale pour haute trahison, espionnage et collaboration active avec l'Allemagne ennemie, de décembre 1917 à février $1918^{(60)}$.

Les royalistes de Görlitz rédigèrent aussi des tracts favorables à l'Allemagne. Ces tracts furent lancés par avion sur le front macédonien durant l'été 1918. Rédigés en grec et adressés aux soldats grecs, ils faisaient d'Élefthérios Venizélos un grand ennemi du pays et de Constantin un roi bassement séparé de son peuple bien-aimé avec lequel il avait triomphé dans les deux guerres balkaniques. Ils appelaient ainsi les combattants grecs à suivre l'exemple du IV corps d'armée en se rendant aux Allemands ${ }^{(61)}$.

Sur un autre plan, toujours pour soutenir leur roi bien-aimé et l'Allemagne, les officiers royalistes se montrèrent prêts à combattre en Épire ou ailleurs les vénizélistes ${ }^{(62)}$. Une telle campagne vers les Balkans semble même s'être discutée entre le roi Constantin et les officiers grecs en novembre 1917 en Suisse ${ }^{(63)}$. L'entourage du roi envisagea également de placer le IV e corps d'armée aux côtés des troupes allemandes dans les Balkans, ce qui fut proposé aux Allemands ${ }^{(64)}$. Enfin, aucune opération des "prisonniers" grecs contre leurs compatriotes n'eut lieu alors que les gendarmes vénizélistes refusèrent de faire un pas vers le sud comme leur officier Ioannis Papadakis, avant son transfert en Westphalie ${ }^{(65)}$, le leur avait fermement ordonné(66).

Finalement l'armistice de novembre 1918 permit la libération des troupes grecques d'Allemagne mais avec difficulté. Les officiers royalistes refusèrent en effet de laisser rentrer en Grèce les soldats et les gendarmes avant que le gouvernement vénizéliste

58 Voir aussi G. Alexatos, Les Grecs (note 20), p. 163.

59 Musée Benakis, Archives d'Élefthérios Venizélos, Enveloppe 14, Télégramme du consul grec à Bern Markettis adressé au consul grec de Paris, Berne, 30 novembre 1917, p. 1.

60 Pour plus d'informations sur cette affaire, voir le journal Empros entre le 13 et le 17 mars 1918.

61 Les manifestes sont présentés à la cour martiale, juin 1920. «Le procès de Görlitz », Macédoine, 9/2996 (10 juin 1920), p. 2.

62 «Comment ont vécu les soldats grecs à Görlitz», Macédoine, 8/2574 (18 mars 1919), p. 1; Musée Benakis, Archives d'Élefthérios Venizélos, Enveloppe 354, Rapport d’Ioannis Papadakis quant à la vie des vénizélistes à Görlitz, Athènes, 14 février 1919, p. 5.

63 Musée Benakis, Archives d'Élefthérios Venizélos, Enveloppe 14, Télégramme du consul grec à Bern Markettis adressé au consul grec de Paris, Berne, 30 novembre 1917, p. 1.

64 Sur ces tentatives des royalistes de Suisse, voir aussi G. Alexatos, Les Grecs (note 20), p. 167-168.

65 Musée Benakis, Archives d’Élefthérios Venizélos, Enveloppe 354, Rapport d'Ioannis Papadakis quant à la vie des vénizélistes à Görlitz, Athènes, 14 février 1919, p. 5.

66 Ibid.; «Comment ont vécu les soldats grecs à Görlitz», Macédoine, 8/ 2574 (18 mars 1919), p. 1. 
ne signât le décret d'amnistie pour tous les officiers de Görlitz, ce qui provoqua une révolte et le départ spontané d'Allemagne du plus grand nombre. En l'espace de quelques jours, Görlitz se vida mais le retour en Grèce de tous les invités du Kaiser n'eut lieu qu'en février 1919, via Fiume, Varna et Costanza ${ }^{(67)}$. Les officiers enfermés en Westphalie, quant à eux, prirent la route vers la Grèce le 27 décembre 1918. Passant par Düsseldorf et traversant la Belgique, les trente-six officiers grecs gagnèrent Paris, accueillis par Élefthérios Venizélos. Ils y restèrent pour plusieurs jours avant de partir pour Marseille le 9 janvier 1919. Fin janvier 1919, ils débarquèrent enfin dans le port de Salonique ${ }^{(68)}$.

Dès lors, la Discorde nationale devint de nouveau une affaire uniquement grecque et la période «internationale» de l'affaire finit en juillet 1920. La cour martiale condamna à mort, en effet, huit officiers grecs pour avoir permis cette «capture» considérée comme honteuse ${ }^{(69)}$; le reste des officiers royalistes, acquittés de leurs actes et de leur attitude à Görlitz, fut réformé de l'armée grecque ${ }^{(70)}$.

\title{
Conclusion
}

En définitive, la Discorde nationale constitua l'une des crises politiques les plus graves de l'histoire grecque. Éclatant au cours de la Grande Guerre à cause de l'opposition du roi Constantin et d'Élefthérios Venizélos sur la participation ou non de leur pays à la guerre, elle dépassa les frontières de l'État grec. Par l'intermédiaire des troupes grecques «capturées» en Macédoine orientale en octobre 1916 et par le biais du roi Constantin expulsé de Grèce en juin 1917, cette crise grecque impliqua des autorités et des peuples étrangers. Mais, une fois la guerre finie, cette Discorde nationale redevint une affaire purement grecque qui continua à polluer la vie politique du pays pendant bien des années encore.

\section{Résumé}

Cet article vise à montrer de quelle manière une crise politique majeure de Grèce, la Discorde nationale, se diffusa au-delà des frontières du pays et impliqua des autorités étrangères. Par le biais, d'un côté, des soldats grecs "capturés" par des troupes bulgaroallemandes en septembre 1916 et, de l'autre, de la famille royale expulsée de Grèce en juin 1917, la Discorde nationale occupa l'Europe, où elle se transforma en guerre civile.

\begin{abstract}
This paper aims to present how a great political crisis of Greece during the Great War, the National Schism, was spread beyond the Greek borders and made foreign authorities
\end{abstract}

67 Au sujet du retour en Grèce, voir G. Alexatos, Les Grecs (note 20), p. 193-216. Certes, parmi les troupes grecques, il y avait aussi des «capturés» qui sont restés pour toujours en Allemagne. Ibid., p. 221-225.

68 Alexatos/Dordanas/Kandylakes (éd.), À Görlitz (note 26), p. 117-121.

69 G. Alexatos, Les Grecs (note 20), p. 206-211. Voir aussi la presse grecque entre le 9 mai et le 29 juin 1920.

70 Ibid. 
to be implicated. The Greek soldiers captured by Bulgarian and German military forces in September 1916 transmitted the National Schism in Europe. King Constantine expelled from Greece in June 1917 contributed to this diffusion as well. Finally, in the heart of Europe, the National Schism has been transformed into a civil war. 